\title{
NEMO 1-D: the first NEGF-based TCAD tool
}

\author{
Gerhard Klimeck \\ Purdue University, School of Electrical and Computer Engineering, \\ Network for Computational Nanotechnology, West Lafayette, IN 47906 \\ Jet Propulsion Laboratory, California Institute of Technology, Pasadena, CA 91109 \\ email: gekco_at_purdue_edu
}

\begin{abstract}
The fundamentally sound non-Equilibrium Green's function (NEGF) approach provides the theoretical basis for NEMO 1-D as the first nanoelectronic TCAD tool. Effects of quantum charging, bandstructure and incoherent scattering from alloy disorder, interface roughness, acoustic phonons, and polar optical phonons are modeled. Engineers and experimentalists who desire a black-box design tool as well as theorists who are interested in a detailed investigation of the physics have found NEMO useful. Access to this comprehensive theoretical framework is accommodated by a graphical user interface (GUI) which configures the usage of a collection of models that trade off physical content with speed and memory requirements. This article describes the NEMO origin, provides modern references to NEGF, accumulates the diverse references to the NEMO results, and provides a perspective on NEGF in future TCAD tools.
\end{abstract}

\section{History and Acknowledgements}

The nanoelectronic modeling (NEMO) program is the result of a multi-year development effort involving an industrial research laboratory (Central Research Laboratory of Texas Instruments, later transferred to Raytheon TI Systems), several Universities (UT Dallas, Ohio State U., U. Alabama in Huntsville), and a US Federally Funded Development and Research Center (NASA, JPL, California Institute of Technology). The original development began in 1993 with the lead of TI and concluded with the delivery of the tool to the US government sponsor in January of 1998. The development then shifted to the NASA Jet Propulsion Laboratory.

A large number of people involved with the NEMO 1-D code over the past 11 years. I would especially like to acknowledge the key contributors to the development of the tool: Drs. Roger Lake, R. Chris Bowen, Dan Blanks, Bill Frensley, and Tim Boykin. The primary application focus was the quantitative modeling of resonant tunneling diodes (RTDs). These III-V high speed devices were developed for high speed applications such as analog-digital-converters [1] and memory devices [2].

\section{Modern NEGF Method References for Carrier Transport}

Novel aspects of the theory developed under the NEMO program are described in detail in [3]. Early work on the NEGF approach has been focussed on bulk many-body interactions and the literature was difficult to penetrate. Just over the past few years Datta has published $[4,5]$ introductions to NEGF that enable the electrical engineer to 
explore and adopt the approach. Also Lake has published an overview on NEGF in 2D and 3D transport [6]. The Network for Computational Nanotechnology is the host of the nanoHUB [7], where students, researchers, and teachers can use on-line simulation tools for free. Datta's course "From Atoms to Transistors" is available [8] for free on the nanHUB and more NEGF material will be posted there shortly.

\section{The NEMO 1-D Simulation Tool}

Simulation of realistic extended devices: A variety of different growth and deposition techniques opened the design space for a large variety of devices. The design of RTDs requires accurate modeling of the quantum charge, resonant levels, and scattering effects in spatially complicated varying potential profiles. The quantitative and experimentally relevant modeling of realistic extended RTDs poses two great theoretical and numerical challenges: the inclusion of band-structure and scattering effects in realisticly extended devices (see for example Figs. (1) and (2) of reference [9]). The formulation of the problem using the NEGF approach enabled the derivation of new boundary conditions. These boundary conditions enable the modeling of the carrier injection from quasi-bound emitter states in the same footing with continuum emitter states. Chargeself-consistent band-bending in the reservoir regions surrounding the central RTD is included. The boundary conditions are demonstrated in $[9,10,11,12,13]$.

Multi-band Models for High Performance RTDs: The importance of full-bandstructure calculations for InGaAs / InAlAs RTDs are described in [12, 11, 13]. Interband tunneling in $\mathrm{Si} / \mathrm{SiO}_{2} /$ Metal structures is calculated in [14]. Boykin's development of new sp3s* tight binding material parameters $[15,16]$ was essential in the quantitative simulation of RTDs at high temperatures.

Incoherent Scattering: Quantitative comparisons between the experimental and calculated valley current of an RTD are made in [17, 18, 19]. Ref. [17] compared RTD currents using different interface roughness models for the first time. Ref. [12] demonstrates the first calculation in which scattering assisted charging and Poisson's equation were calculated self-consistently in a fully quantum-mechanical model.

As a bottom line one can state that much to our surprise, the development of the extended boundary conditions and full-bandstructure simulation was the key to the quantitative simulation of high performance, high current density, room temperature conduction band RTDs. The essential valley current generation mechanism is really transport through excited states that are only accurately prediced when band non-parabilicity, and band warping is properly included. The approximate modeling of the strong scattering in the emitter contact is absolutely essential. Scattering in the central region due to polar optical phonons and interface roughness scattering turns out to be really only important at low temperatures for typical RTDs.

Software Technology: Resonance finding and grid generation algorithms [20, 21] were developed to efficiently integrate rapidly varying spectral functions with a minimal number of computational nodes. The NEMO software is designed in an object oriented approach in C, with numerical intensive algorithms in F90, and a graphical user interface in X11/Motif [22]. It consists roughly of 250,000 lines of source code. It can be operated in batch and GUI mode.

NEMO Extensions at JPL: At JPL NEMO was extended with several independent developments. The software was parallelized using MPI on various loop levels (bias 
points, momentum grid, and energy grid) [23]. It can now run efficiently on various parallel machines including commodity clusters. While typical runs with NEMO simply require a normal workstation, the exploration of details of momentum dependent transport of holes [24] and electrons [25] benefited greatly from parallelization. To enable simulation of higher resistivity structures and to explore the validity of the reservoir assumption a drift diffusion models was integrated into NEMO [26]. Applicability of NEMO to model cascade lasers was explored in reference [27].

Devices Synthesis: Finally NEMO was embedded inside a genetic algorithm (GA) package which can drive design parameters such as heterostructure thicknesses and doping profiles to a desired device performance [28]. This GENES package (Genetically Engineered Nanoelectronic Structures) enables device synthesis and characterization. The GA extension has also been used extensively to optimize tight binding parameters $[29,30]$.

NEMO 3-D has been developed [31] at JPL for the calculation of electronic structure in quantum dots consistent of the explicit representation of millions of atoms.

\section{NEGF and other Simulators}

NEMO has proven to be a standard for RTD simulations. NEMO has been used to benchmark Wigner function [32] and quantum hydrodynamic [33] approaches. These approaches are developed with the motivation to simulate quantum effects rapidly. However, do-date the NEGF approach appears to be significantly more stable and faster for the modeling of RTDs with realistic barrier heights, widths, and material dependent effective masses. Also these approaches have not yet begun to address multiband transport issues, that were identified as essential.

A subset of NEMO 1-D has been implemented in the software WinGreen [34]. Others have proceeded to develop 1-D approaches to scattering in a multi-band basis for Si interband processes [35, 36] and general RTD scattering [37]. Lake also begun development of a full band 3-D NEGF model [38, 39]. Anantram, Datta, Lundstrom, Jovanovic, and Ogawa have pushed forward NEGF device simulation for ultra-scaled CMOS devices where a 2-D spatial domain is resolved [40, 41, 6, 42].

NEGF usage is also expanding into the novel field of molecular electronics [43, 5] and even software has emerged on the commercial market [44].

\section{NEMO Availability}

The NEMO 1-D transport code is currently not being distributed anymore by the commercial owner Raytheon. U.S. Government use licenses can be extended by JPL/Caltech. Please contact Gerhard Klimeck on this matter by email at gekco_at_jpl_nasa_gov. There are on-going discussions at this time to provide a better code release mechanism. NEMO 3-D which currently is a pure electronic structure code [31] is available freely in its alpha version on http://www.openchannelsoftware.com/projects/NEMO_3D.

\section{References}

[1] T. P. E. Broekaert et al., 33, 1342 (1998).

[2] J. P. A. van der Wagt, A. C. Seabaugh, and E. Beam III, 19, 7 (1998).

[3] R. Lake, G. Klimeck, R. C. Bowen, and D. Jovanovic, J. Appl. Phys. 81, 7845 (1997).

[4] S. Datta, in IEDM (IEEE, NY, 2002). 
[5] S. Datta, Nanotechnology 15, S433 (2004).

[6] R. Lake et al. http://www.ee.ucr.edu/rlake/dresden02_final_unix.pdf.

[7] http://nanohub.org - A community resource for On-line simulation and more.

[8] Supriyo Datta, on-line course, "Quantum Phenomena: From Atoms to Transistors", http://www.nanohub.org/quantum_phenomena.

[9] G. Klimeck et al., Appl. Phys. Lett. 67, 2539 (1995).

[10] G. Klimeck et al. in the 1995 Ann. Dev. Res. Conf. Digest, (IEEE, NJ, 1995), p. 52.

[11] R. C. Bowen et al., J. Appl. Phys 81, 3207 (1997).

[12] R. Lake et al. in the 1996 Ann. Dev. Res. Conf. Digest, (IEEE, NJ, 1996), p. 174.

[13] G. Klimeck et al. in the 1997 Ann. Dev. Res. Conf. Digest, (IEEE, NJ, 1997), p. 92.

[14] C. Bowen et al., in IEDM 1997 (IEEE, New York, 1997), pp. 869-872.

[15] T. B. Boykin, Phys. Rev. B 54, 8107 (1996).

[16] T. Boykin et al., submitted to Phys. Rev. B (1997).

[17] R. Lake et al., Superlatt. and Microstruct. 20, 279 (1996).

[18] G. Klimeck et al., in Quantum Devices and Circuits, edited by K. Ismail, S. Bandyopadhyay, and J. P. Leburton (Imperial College Press, London, 1997), pp. 154-159.

[19] G. Klimeck et al., Phys. Stat. Sol. (b) 204, 408 (1997).

[20] R. C. Bowen, W. R. Frensley, G. Klimeck, and R. Lake, PRB 52, 2754 (1995).

[21] G. Klimeck et al., VLSI Design 6, 107 (1998).

[22] G. Klimeck et al., VLSI Design 8, 79 (1998).

[23] G. Klimeck, J. of Computational Electr. 1, 75 (2002).

[24] G. Klimeck, R. C. Bowen, and T. B. Boykin, Phys. Rev. B 63, 195310 (2001).

[25] G. Klimeck, Physica Status Solidi (b) 226, 9 (2001).

[26] G. Klimeck, J. of Computational Electr. 2, 177 (2003).

[27] J. Green et al., in CLEO/Europe 2003 (CLEO, Munich, 2003).

[28] G. Klimeck, C. H. Salazar-Lazaro, A. Stoica, and T. Cwik, in Proc. Mat. Res. Soc. (Mat. Res. Soc., Boston, 1998), Vol. 559, p. 149.

[29] G. Klimeck et al., Superl. and Microstr. 27, 77 (2000).

[30] T. B. Boykin, G. Klimeck, and F. Oyafuso, Phys. Rev. B. 69, 115201 (2004).

[31] G. Klimeck et al., Comp. Modeling in Eng. and Sci. (CMES) 3, 601 (2002).

[32] H. Kosina, G. Klimeck, M. Nedjalkov, and S. Selberherr, in SISPAD 2003 (IEEE, Boston, 2003), pp. 171-174.

[33] C. L. Gardner, G. Klimeck, and C. Ringhofer, subm. to J. of Comp. Electr. (2004).

[34] http://www.fz-juelich.de/isg/mbe/software.html.

[35] C. Rivas et al., Appl. Phys. Lett. 78, 814 (2001).

[36] C. Rivas et al., J. of Appl. Phys 94, 5005 (2003).

[37] R. T. T. M. Matsuto Ogawa, Takashi Sugano, Physica B 272, 167 (1999).

[38] C. Rivas and R. Lake, phys. stat. sol (a) 195, 3 (2003).

[39] C. Rivas and R. Lake, (Nanotech, San Francisco, 2003), Vol. 559, p. 149.

[40] Z. Ren et al., IEEE T Electr. Dev. 50, 12 (2003).

[41] A. Svizhenko and M. Anantram, IEEE T. Electr. Dev 50, 8 (2003).

[42] M. Ogawa, H. Tsuchiya, and T. Miyoshi, IEEE SISPAD 261 (2002).

[43] P. Damle, A. Ghosh, and S. Datta, Phys. Rev.B, Rap. Comm. 201403 (2001).

[44] M. Brandbyge et al., Phys. Rev. B. 65, 165401 (2002). 